Article

\title{
An Application of Equivalence Transformations to Reaction Diffusion Equations
}

\author{
Mariano Torrisi $^{\dagger}{ }^{\dagger} *$ and Rita Tracinà ${ }^{\dagger}$ \\ Department of Mathematics and Computer Sciences, University of Catania, Catania I 95125, Italy; \\ E-Mail: tracina@dmi.unict.it \\ ${ }^{\dagger}$ These authors contributed equally to this work. \\ * Author to whom correspondence should be addressed; E-Mail: torrisi@ dmi.unict.it or \\ m.torrisi12@gmail.it.
}

Academic Editor: Roman M. Cherniha

Received: 15 July 2015 / Accepted: 15 October 2015 / Published: 23 October 2015

\begin{abstract}
In this paper, we consider a quite general class of advection reaction diffusion systems. By using an equivalence generator, derived in a previous paper, the authors apply a projection theorem to determine some special forms of the constitutive functions that allow the extension by one of the two-dimensional principal Lie algebra. As an example, a special case is discussed at the end of the paper.
\end{abstract}

Keywords: equivalence transformations; groups of transformations; classical symmetries; biomathematical models

\section{Introduction}

In this paper, we focus our attention on the following family of $2 \times 2$ nonlinear advection reaction diffusion systems in $(1+1)$ independent variables:

$$
\left\{\begin{array}{l}
u_{t}=\left(f(u) u_{x}\right)_{x}+g\left(u, v, u_{x}\right) \\
v_{t}=h(u, v)
\end{array}\right.
$$

with $f(u), g\left(u, v, u_{x}\right), h(u, v)$ analytic functions. These systems, apart from their own mathematical interest, offer the possibility to be analyzed as possible biomathematical models for two interacting 
species $u$ and $v$, where one of them, the species $v$, does not suffer diffusion. The dependence of the function $g$ on the gradient $u_{x}$ shows advection effects; in fact, the individuals of the species $u$ could be influenced by external stimuli as wind velocity or water currents. Of course, the absence of the advective phenomena brings to the following system:

$$
\left\{\begin{array}{l}
u_{t}=\left(f(u) u_{x}\right)_{x}+g(u, v), \\
v_{t}=h(u, v),
\end{array}\right.
$$

that can describe the evolution of the Aedes aegypti mosquito population in a region where wind effects are negligible or the evolution of a Proteus mirabilis bacterial colony when the diffusion coefficient depends only on the species $u$, that is when the system (2) is a subclass of the following wider class:

$$
\left\{\begin{array}{l}
u_{t}=\left(f(u, v) u_{x}\right)_{x}+g(u, v), \\
v_{t}=h(u, v)
\end{array}\right.
$$

considered in [1-4].

One of the most important problems in modeling the phenomena of life sciences and natural sciences is to select "good" forms of arbitrary functions (constitutive equations) that fit well with the experimental data and possess mathematical properties that allow scientists to get some solutions or much news about them.

A powerful tool of investigation in this field is given from transformation groups, in particular from equivalence transformations and symmetries.

In the framework of the group analysis, the literature concerning the systems of the type (1) is scarce. There are no papers devoted to a complete Lie symmetry analysis of PDE systems with advection (convection) terms of the form (1). In [5], it is possible to find a complete solution of this problem for a class of diffusion systems with convection terms in both equations. Moreover, the paper [6] contains some description of Lie symmetries for a class of systems, which includes cases having a structure similar to system (3). However, it is possible to find some papers devoted to the complete Lie symmetry analysis of a single advection (convection) reaction diffusion equation (see, e.g., [7-9]).

Following, e.g., [10], an equivalence transformation for the system (1) is a non-degenerate change of the independent and dependent variables $t, x, u, v$ into $\hat{t}, \hat{x}, \hat{u}, \hat{v}$ :

$$
\left\{\begin{array}{l}
x=x(\hat{x}, \hat{t}, \hat{u}, \hat{v}), \\
t=t(\hat{x}, \hat{t}, \hat{u}, \hat{v}) \\
u=u(\hat{x}, \hat{t}, \hat{u}, \hat{v}) \\
v=v(\hat{x}, \hat{t}, \hat{u}, \hat{v})
\end{array}\right.
$$

that transforms a system of the class (1) in another one of the same class. That is, an equivalence transformation brings the system of the form (1) in a system preserving the differential structure, but, in general, with:

$$
\hat{f}(\hat{u}) \neq f(u), \quad \hat{g}\left(\hat{u}, \hat{v}, \hat{u}_{\hat{x}}\right) \neq g\left(u, v, u_{x}\right), \quad \hat{h}(\hat{u}, \hat{v}) \neq h(u, v) .
$$

It maps a solution of a system in a solution of the equivalent system. 
It could occur that the transformed equations show still the same structure, but the arbitrary functions are depending on additional variables. In this case, the equivalence is said weak.

Of course, in the case:

$$
\hat{f}(\hat{u})=f(u), \quad \hat{g}\left(\hat{u}, \hat{v}, \hat{u}_{\hat{x}}\right)=g\left(u, v, u_{x}\right), \quad \hat{h}(\hat{u}, \hat{v})=h(u, v),
$$

an equivalence transformation becomes a symmetry (a transformation of variables that leaves invariant the transformed system).

A symmetry allows one to reduce the number of independent variables of an equation so that, for instance, a PDE in $1+1$ independent variables can become an ODE. Once solved this last one, going back to the original variables, we get a solution that is invariant with respect to the symmetries used for the reduction. It is worthwhile to note that a symmetry transforms invariant solutions into invariant solutions that are not essentially different (see Ovsiannikov [11]), but, having a different form, they could satisfy different suitable initial/boundary conditions.

The aim of this paper is an improvement of the results that we have shown in [12], bearing in mind some generalization of the special form assumed from the constitutive functions $f, g$ and $h$ already used in some previous papers about $[4,12-14]$. In this paper, we use the infinitesimal generator of equivalence transformations derived in [12] for the class (1) in order to obtain some extensions of the principal Lie algebra for the following subclass:

$$
\begin{aligned}
& u_{t}=\left(f(u) u_{x}\right)_{x}+u^{r} u_{x}+\Gamma_{1}(u)+\Gamma_{2}(v), \\
& v_{t}=h(u, v)
\end{aligned}
$$

Here, we assumed:

$$
g\left(u, v, u_{x}\right)=u^{r} u_{x}+\Gamma_{1}(u)+\Gamma_{2}(v)
$$

that is a generalization of that ones used in $[12,13]$.

In the next section, after recalling, for the sake of completeness, some elements about equivalence transformations (for additional mathematical and methodical details, the interested reader can see $[12,15,16])$, we write the equivalence generator derived in [12]. In Section 3, the principal Lie algebra and its extensions are discussed; moreover, a simple example that could be related to the biomathematical model of Aedes aegypti is considered. The conclusions are given in the last section.

\section{On Equivalence Transformations and Their Calculation for the Class (1)}

It is easy to ascertain that, often, in papers on differential equations, it is possible to find several examples of equivalence transformations and their applications. In general, in the past and now, the direct search for the most general equivalence transformations through the finite form of the transformation has been used. Quite often, this search is connected to considerable computational difficulties and does not always lead to the complete solution of the problem (e.g., $[17,18]$ ). 


\subsection{Elements on Equivalence Transformations}

Following [11,15,16,19,20] (see also, e.g., [10,21-23]), we look for the infinitesimal generator of the equivalence transformations of the system (1) of the form:

$$
Y=\xi^{1} \partial_{x}+\xi^{2} \partial_{t}+\eta^{1} \partial_{u}+\eta^{2} \partial_{v}+\mu^{1} \partial_{f}+\mu^{2} \partial_{g}+\mu^{3} \partial_{h}
$$

where the infinitesimal components $\xi^{1}, \xi^{2}, \eta^{1}, \eta^{2}$ are sought depending on $x, t, u$, $v$, while the infinitesimal components $\mu^{i}(i=1,2,3)$ can also depend on $u_{t}, u_{x}, v_{t}, v_{x}, f, g$ and $h$. Here, we are interested in obtaining the infinitesimal coordinates $\xi^{i}, \eta^{i}$ and $\mu^{j}(i=1,2$ and $j=1,2,3)$, by applying the Lie-Ovsiannikov infinitesimal criterion [11] by requiring the invariance, with respect to a suitable prolongations $Y^{(1)}$ and $Y^{(2)}$ of generator (9), of the following equations:

$$
\begin{aligned}
& u_{t}-\left(f u_{x}\right)_{x}-g=0, \\
& v_{t}-h=0
\end{aligned}
$$

without requiring the invariance of the so-called auxiliary conditions $[15,16,24,25]$ :

$$
\begin{gathered}
f_{t}=f_{x}=f_{v}=f_{u_{x}}=f_{u_{t}}=f_{v_{x}}=f_{v_{t}}=g_{t}=g_{x}=g_{u_{t}}=g_{v_{t}}=g_{v_{x}}=0, \\
h_{t}=h_{x}=h_{u_{x}}=h_{u_{t}}=h_{v_{x}}=h_{v_{t}}=0,
\end{gathered}
$$

that characterize the functional dependence of $f, g$ and $h$.

In this way, we obtain the weak equivalence transformations $[15,16]$.

The main difference with respect to the classical one is that the infinitesimal operators of weak equivalence transformations can generate transformations that do not preserve the functional dependence of the arbitrary elements.

With respect to the application in biomathematical models, equivalence and weak equivalence transformations were applied not only to study tumor models [26,27], but also the population dynamics in $[1,3,4]$.

\subsection{Calculation of Weak Equivalence Transformations}

We need the following prolongations $Y^{(1)}$ and $Y^{(2)}$ :

$$
\begin{aligned}
Y^{(1)} & =Y+\zeta_{1}^{1} \partial_{u_{x}}+\zeta_{2}^{1} \partial_{u_{t}}+\zeta_{1}^{2} \partial_{v_{x}}+\zeta_{2}^{2} \partial_{v_{t}}+\omega_{u}^{1} \partial_{f_{u}} \\
Y^{(2)} & =Y^{(1)}+\zeta_{x x}^{1} \partial_{u_{x x}}
\end{aligned}
$$

with (see [12] for more details),

$$
\begin{aligned}
& \zeta_{1}^{1}=D_{x} \eta^{1}-u_{x} D_{x} \xi^{1}-u_{t} D_{x} \xi^{2}, \\
& \zeta_{2}^{1}=D_{t} \eta^{1}-u_{x} D_{t} \xi^{1}-u_{t} D_{t} \xi^{2}, \\
& \zeta_{1}^{2}=D_{x} \eta^{2}-v_{x} D_{x} \xi^{1}-v_{t} D_{x} \xi^{2}, \\
& \zeta_{2}^{2}=D_{t} \eta^{2}-v_{x} D_{t} \xi^{1}-v_{t} D_{t} \xi^{2} \\
& \zeta_{11}^{1}=D_{x} \zeta_{1}^{1}-u_{x x} D_{x} \xi^{1}-u_{t x} D_{x} \xi^{2} \\
& \omega_{u}^{1}=\tilde{D}_{u} \mu^{1}-f_{u} \tilde{D}_{u} \eta^{1}
\end{aligned}
$$


where $D_{x}$ and $D_{t}$ are, respectively, the total derivatives with respect to $x$ and $t$, while in our case, the operator $\tilde{D}_{u}$ is defined as:

$$
\tilde{D}_{u}=\partial_{u}+f_{u} \partial_{f}+g_{u} \partial_{g}+h_{u} \partial_{h}
$$

The invariant conditions read:

$$
\begin{aligned}
& \zeta_{2}^{1}-2 \zeta_{1}^{1} u_{x} f_{u}-u_{x}^{2} \omega_{u}^{1}-u_{x x} \mu^{1}-f \zeta_{11}^{1}-\mu^{2}=0 \\
& \zeta_{2}^{2}-\mu^{3}=0
\end{aligned}
$$

both under the constraints (10) and (11).

Following the usual techniques, we derive the following infinitesimal components for the weak equivalence generators:

$$
\begin{aligned}
& \xi^{1}=\alpha(x), \quad \xi^{2}=\beta(t), \quad \eta^{1}=\delta(t, u), \quad \eta^{2}=\lambda(x, t, v), \\
& \mu^{1}=\left(2 \alpha^{\prime}-\beta^{\prime}\right) f, \quad \mu^{2}=\delta_{t}+\left(\delta_{u}-\beta^{\prime}\right) g+\left(\alpha^{\prime \prime} u_{x}-\delta_{u u} u_{x}^{2}\right) f, \quad \mu^{3}=\left(\lambda_{v}-\beta^{\prime}\right) h+\lambda_{t},
\end{aligned}
$$

where $\alpha(x), \beta(t), \delta(t, u), \lambda(x, t, v)$ are arbitrary real functions of their arguments. The corresponding infinitesimal generator is:

$$
\begin{aligned}
Y & =\alpha(x) \partial_{x}+\beta(t) \partial_{t}+\delta(t, u) \partial_{u}+\lambda(x, t, v) \partial_{v}+\left(2 \alpha^{\prime}-\beta^{\prime}\right) f \partial_{f} \\
& +\left(\delta_{t}+\left(\delta_{u}-\beta^{\prime}\right) g+\left(\alpha^{\prime \prime} u_{x}-\delta_{u u} u_{x}^{2}\right) f\right) \partial_{g}+\left(\left(\lambda_{v}-\beta^{\prime}\right) h+\lambda_{t}\right) \partial_{h}
\end{aligned}
$$

\section{Symmetries for a Subclass of Advection Reaction Diffusion Systems}

In this section, we apply the projection theorem, introduced in [28] and successively generalized in $[15,16,24]$, in order to carry out a symmetry classification for the following subclass of system (1):

$$
\begin{aligned}
& u_{t}=\left(f(u) u_{x}\right)_{x}+u^{r} u_{x}+\Gamma_{1}(u)+\Gamma_{2}(v), \\
& v_{t}=h(u, v),
\end{aligned}
$$

with $r \neq 0, \Gamma_{1}^{\prime} \neq 0$ and $\Gamma_{2}^{\prime} \neq 0$.

For the system (28), we can affirm the following:

Theorem 1. The projection of the infinitesimal weak equivalence generator $Y$ for the system (1) on the space $(x, t, u, v)$ :

$$
X=\alpha(x) \partial_{x}+\beta(t) \partial_{t}+\delta(t, u) \partial_{u}+\lambda(x, t, v) \partial_{v}
$$

is an infinitesimal symmetry generator of a system of the class (28) if and only if the constitutive equations, specifying the forms of $f, g$ and $h$, are invariant with respect to $Y$.

Applying the previous theorem, in order to obtain the determining system for the subclass (28), we request the invariance with respect to $Y$ of the following constitutive equations:

$$
\begin{aligned}
& f=f(u), \\
& g=u^{r} u_{x}+\Gamma_{1}(u)+\Gamma_{2}(v), \\
& h=h(u, v),
\end{aligned}
$$


that is

$$
\begin{aligned}
Y(f-f(u)) & =0, \\
Y\left(g-u^{r} u_{x}-\Gamma_{1}(u)-\Gamma_{2}(v)\right) & =0, \\
Y(h-h(u, v)) & =0,
\end{aligned}
$$

under the constraints (30). Then, taking into account the form (27) of generator $Y$, we have the following determining equations:

$$
\begin{aligned}
& \left(2 \alpha^{\prime}-\beta^{\prime}\right) f-\delta f_{u}=0, \\
& \delta_{t}+\left(\delta_{u}-\beta^{\prime}\right)\left(u^{r} u_{x}+\Gamma_{1}+\Gamma_{2}\right)-\left(\delta_{u u} u_{x}^{2}-\alpha^{\prime \prime} u_{x}\right) f-\left(\delta_{u}-\alpha^{\prime}\right) u_{x} u^{r}- \\
& \quad \delta\left(r u^{r-1} u_{x}+\Gamma_{1}{ }^{\prime}\right)-\lambda \Gamma_{2}{ }^{\prime}=0 \\
& \left(\lambda_{v}-\beta^{\prime}\right) h+\lambda_{t}-\delta h_{u}-\lambda h_{v}=0 .
\end{aligned}
$$

We recall here that the principal Lie algebra $L_{\mathcal{P}}[10,19]$ is the Lie algebra that leaves invariant the system (28) for any form of the functions $f(u), \Gamma_{1}(u), \Gamma_{2}(v)$ and $h(u, v)$. Then, the principal Lie algebra is the generator (29) where the functions $\alpha, \beta, \delta$ and $\lambda$ are solutions of the system (32)-(34) for arbitrary functions $f(u), \Gamma_{1}(u), \Gamma_{2}(v)$ and $h(u, v)$. Consequently, it is a simple matter to ascertain the following:

Corollary 2. The projection (29) of infinitesimal weak equivalence generator $Y$ for the system (1) on the space $(x, t, u, v)$ is the infinitesimal symmetry generator corresponding to the principal Lie algebra of the class (28) if and only if $\eta^{i}=0, \mu^{j}=0, i=1,2, j=1,2,3$.

Then, the principal Lie algebra $L_{\mathcal{P}}$ is spanned by the following generators corresponding respectively to translations in time and in the space:

$$
X_{1}=\partial_{t}, X_{2}=\partial_{x}
$$

\subsection{On the Extensions of the $L_{\mathcal{P}}$}

Here, we analyze some particular cases of the extension of the principal algebra for the class (28). That is, we look for a family of particular functions $f(u), \Gamma_{1}(u), \Gamma_{2}(v)$ and $h(u, v)$, such that the solution of system (32)-(34) is different from $\delta=\lambda=0$ and $\alpha$ and $\beta$ constants, which corresponds to the generators (35).

From Equation (32), deriving with respect to $x$, we get:

$$
2 \alpha^{\prime \prime} f=0
$$

that is

$$
\alpha(x)=a_{1} x+a_{0},
$$

with $a_{0}$ and $a_{1}$ arbitrary constants. Consequently, from Equation (33), deriving with respect to $x$, we get:

$$
-\lambda_{x} \Gamma_{2}{ }^{\prime}=0
$$


and taking into account that $\Gamma_{2}^{\prime} \neq 0$, we obtain:

$$
\lambda=\lambda(t, v)
$$

Equation (33) becomes:

$$
\delta_{t}+\left(\delta_{u}-\beta^{\prime}\right)\left(\Gamma_{1}+\Gamma_{2}\right)-\delta \Gamma_{1}{ }^{\prime}-\lambda \Gamma_{2}{ }^{\prime}+u_{x}\left(\left(a_{1}-\beta^{\prime}\right) u^{r}-\delta r u^{r-1}\right)+u_{x}^{2}\left(-\delta_{u u} f\right)=0 .
$$

As any function does not depend on $u_{x}$, from Equation (40), we derive:

$$
\begin{aligned}
& \delta_{u u} f=0, \\
& \left(a_{1}-\beta^{\prime}\right) u^{r}-\delta r u^{r-1}=0, \\
& \delta_{t}+\left(\delta_{u}-\beta^{\prime}\right)\left(\Gamma_{1}+\Gamma_{2}\right)-\delta \Gamma_{1}{ }^{\prime}-\lambda \Gamma_{2}{ }^{\prime}=0 .
\end{aligned}
$$

Then, from Equation (41):

$$
\delta(t, u)=u A_{1}(t)+A_{2}(t)
$$

with $A_{1}(t)$ and $A_{2}(t)$ arbitrary functions of $t$. After these partial results, for the sake of clarity, we rewrite the determining system:

$$
\begin{aligned}
& \left(2 a_{1}-\beta^{\prime}\right) f-\left(u A_{1}+A_{2}\right) f_{u}=0, \\
& u\left(a_{1}-\beta^{\prime}-r A_{1}\right)-r A_{2}=0, \\
& \left(u A_{1}{ }^{\prime}+A_{2}{ }^{\prime}\right)+\left(A_{1}-\beta^{\prime}\right)\left(\Gamma_{1}+\Gamma_{2}\right)-\left(u A_{1}+A_{2}\right) \Gamma_{1}{ }^{\prime}-\lambda \Gamma_{2}{ }^{\prime}=0, \\
& \left(\lambda_{v}-\beta^{\prime}\right) h+\lambda_{t}-\left(u A_{1}+A_{2}\right) h_{u}-\lambda h_{v}=0 .
\end{aligned}
$$

From Equation (46), taking into account that any function does not depend on $u$, we get:

$$
A_{1}=\frac{a_{1}-\beta^{\prime}}{r}, \quad A_{2}=0,
$$

then the other equations become:

$$
\begin{aligned}
& \left(2 a_{1}-\beta^{\prime}\right) f-u \frac{a_{1}-\beta^{\prime}}{r} f_{u}=0, \\
& -\frac{\beta^{\prime \prime}}{r} u+\frac{a_{1}-(1+r) \beta^{\prime}}{r}\left(\Gamma_{1}+\Gamma_{2}\right)-u \frac{a_{1}-\beta^{\prime}}{r} \Gamma_{1}^{\prime}-\lambda \Gamma_{2}^{\prime}=0, \\
& \left(\lambda_{v}-\beta^{\prime}\right) h+\lambda_{t}-u \frac{a_{1}-\beta^{\prime}}{r} h_{u}-\lambda h_{v}=0 .
\end{aligned}
$$

We observe that from Equation (50), if $f$ is arbitrary, it follows $\beta=b_{0}, a_{1}=0$, from Equation (51) $\lambda=0$, while the Equation (52) is satisfied. Therefore, for $f$ arbitrary, we do not obtain the extension of the principal Lie algebra. Then, in order to look for extensions of the principal algebra, we observe that from Equation (50), the form of function $f$ must have the following structure:

1. $f=f_{0} u^{r}$.

2. $f=f_{0} u^{s}$ and $s \neq r$.

We study these cases separately. 
1. $f=f_{0} u^{r}$

In this case, from Equation (50), we have $a_{1}=0$. Moreover, by differentiating Equation (51) with respect to $u$, we have:

$$
\beta^{\prime \prime}+\beta^{\prime}\left(r \Gamma_{1}^{\prime}-u \Gamma_{1}^{\prime \prime}\right)=0
$$

We observe that if $\Gamma_{1}$ is arbitrary, we have $\beta=b_{0}$, while from Equation (51), we have $\lambda=0$, and Equation (52) is satisfied; however, we do not obtain the extension of the principal Lie algebra. Then, in order to have extensions of the principal algebra, the following conditions must be satisfied:

$$
\begin{aligned}
& u \Gamma_{1}^{\prime \prime}-r \Gamma_{1}{ }^{\prime}=\gamma_{0}, \\
& \beta^{\prime \prime}=\gamma_{0} \beta^{\prime} .
\end{aligned}
$$

We distinguish two cases: $r \neq-1$ and $r=-1$.

(a) If $r \neq-1$, from Equation (54), we get:

$$
\Gamma_{1}(u)=\frac{c_{1} u^{1+r}}{1+r}-\frac{\gamma_{0} u}{r}+c_{2} .
$$

Consequently, from Equation (51), we obtain:

$$
\lambda(t, v)=-\frac{\left(c_{2}+\Gamma_{2}\right)(r+1) \beta^{\prime}}{r \Gamma_{2}^{\prime}},
$$

while Equation (52) becomes:

$$
\frac{\beta^{\prime}}{r \Gamma_{2}^{\prime 2}} J_{1}=0
$$

with:

$$
J_{1} \equiv h(1+r)\left(c_{2}+\Gamma_{2}\right) \Gamma_{2}^{\prime \prime}-\left(h(1+2 r)-u h_{u}\right) \Gamma_{2}^{\prime 2}+(1+r)\left(h_{v}-\gamma_{0}\right)\left(c_{2}+\Gamma_{2}\right) \Gamma_{2}^{\prime}
$$

We observe that if $\beta^{\prime}=0$, then $\lambda=0$, and we do not obtain the extension of the principal Lie algebra. Consequently, in order to have extensions of the principal algebra, the functions $\Gamma_{2}$ and $h$ must satisfy the equation $J_{1}=0$. In this case, we have two possible generators depending on $\gamma_{0}$.

i. If $\gamma_{0} \neq 0$, as from Equation (55), we have:

$$
\beta(t)=b_{0}+b_{1} e^{\gamma_{0} t}
$$

the additional generator is:

$$
X_{3}=e^{\gamma_{0} t} \partial_{t}-\frac{\gamma_{0} e^{\gamma_{0} t}}{r} u \partial_{u}-\frac{\left(c_{2}+\Gamma_{2}\right)(r+1) \gamma_{0} e^{\gamma_{0} t}}{r \Gamma_{2}^{\prime}} \partial_{v} .
$$


ii. If $\gamma_{0}=0$, as from Equation (55), we have:

$$
\beta(t)=b_{0}+b_{1} t
$$

the additional generator is:

$$
X_{3}=t \partial_{t}-\frac{u}{r} \partial_{u}-\frac{\left(c_{2}+\Gamma_{2}\right)(r+1)}{r \Gamma_{2}^{\prime}} \partial_{v} .
$$

(b) If $r=-1$, from Equation (54), we get:

$$
\Gamma_{1}(u)=c_{1} \ln (u)+\gamma_{0} u+c_{2} .
$$

Consequently, from Equation (51), we obtain:

$$
\lambda(t, v)=-\frac{c_{1} \beta^{\prime}}{\Gamma_{2}^{\prime}},
$$

while Equation (52) becomes:

$$
\frac{\beta^{\prime}}{\Gamma_{2}^{2}} J_{2}=0
$$

with:

$$
J_{2} \equiv h c_{1} \Gamma_{2}^{\prime \prime}-\left(h+u h_{u}\right) \Gamma_{2}{ }^{2}+c_{1}\left(h_{v}-\gamma_{0}\right) \Gamma_{2}{ }^{\prime} .
$$

We observe that if $\beta^{\prime}=0$, then $\lambda=0$, and we do not obtain extension of the principal Lie algebra. Consequently, in order to have extensions of the principal algebra, the functions $\Gamma_{2}$ and $h$ must satisfy the equation $J_{2}=0$. In this case, we have two possible generators depending on $\gamma_{0}$.

i. If $\gamma_{0} \neq 0$, as from Equation (55), we have:

$$
\beta(t)=b_{0}+b_{1} e^{\gamma_{0} t}
$$

the additional generator is:

$$
X_{3}=e^{\gamma_{0} t} \partial_{t}+\gamma_{0} e^{\gamma_{0} t} u \partial_{u}-\frac{c_{1} \gamma_{0} e^{\gamma_{0} t}}{\Gamma_{2}{ }^{\prime}} \partial_{v}
$$

ii. If $\gamma_{0}=0$, as from Equation (55), we have:

$$
\beta(t)=b_{0}+b_{1} t
$$

the additional generator is:

$$
X_{3}=t \partial_{t}-\frac{u}{r} \partial_{u}-\frac{c_{1}}{\Gamma_{2}^{\prime}} \partial_{v} .
$$


2. $f=f_{0} u^{s}$ and $s \neq r$

In this case, from Equation (50), we have:

$$
\beta(t)=\frac{a_{1}(2 r-s)}{r-s} t+b_{0},
$$

and Equation (51) becomes:

$$
a_{1} u \Gamma_{1}{ }^{\prime}-a_{1}(1+2 r-s)\left(\Gamma_{1}+\Gamma_{2}\right)-(r-s) \lambda \Gamma_{2}{ }^{\prime}=0 .
$$

Moreover, by differentiating with respect to $u$, we get:

$$
a_{1}\left(u \Gamma_{1}^{\prime \prime}+(s-2 r) \Gamma_{1}^{\prime}\right)=0 .
$$

We observe that if $\Gamma_{1}$ is arbitrary, then we have $a_{1}=0$, while from Equation (51) $\lambda=0$ and Equation (52) is satisfied, but we do not obtain the extension of the principal Lie algebra. Then, in order to have extensions of the principal algebra, the following condition must be satisfied:

$$
u \Gamma_{1}^{\prime \prime}+(s-2 r) \Gamma_{1}^{\prime}=0 .
$$

We distinguish the following two cases.

(a) If $s \neq 2 r+1$, from Equation (75), we get:

$$
\Gamma_{1}(u)=c_{1}+c_{2} u^{1+2 r-s}
$$

From Equation (51):

$$
\lambda(t, v)=\frac{a_{1}(s-2 r-1)\left(c_{1}+\Gamma_{2}\right)}{(r-s) \Gamma_{2}^{\prime}},
$$

while Equation (52) becomes:

$$
\frac{a_{1}}{(r-s) \Gamma_{2}^{\prime 2}} J_{3}=0
$$

with:

$$
J_{3} \equiv(1+2 r-s)\left(c_{1}+\Gamma_{2}\right)\left(h \Gamma_{2}^{\prime \prime}+h_{v} \Gamma_{2}^{\prime}\right)+\left(u h_{u}-h(1+4 r-2 s)\right) \Gamma_{2}{ }^{2} .
$$

We observe that if the functions $\Gamma_{2}$ and $h$ do not satisfy the equation $J_{3}=0$, we do not obtain the extension of the principal Lie algebra. Then, in order to have extensions of the principal algebra, the functions $\Gamma_{2}$ and $h$ must satisfy the equation $J_{3}=0$. In this case, we obtain the following additional generator:

$$
X_{3}=x \partial_{x}+\frac{2 r-s}{r-s} t \partial_{t}+\frac{1}{s-r} u \partial_{u}+\frac{(s-2 r-1)\left(c_{1}+\Gamma_{2}\right)}{(r-s) \Gamma_{2}^{\prime}} \partial_{v} .
$$


(b) If $s=2 r+1$, from Equation (75), we get:

$$
\Gamma_{1}(u)=c_{1} \ln (u)+c_{2},
$$

and from Equation (72):

$$
\beta(t)=\frac{a_{1}}{r+1} t+b_{0} .
$$

Consequently, from Equation (51), we obtain:

$$
\lambda(t, v)=-\frac{c_{1} a_{1}}{(r+1) \Gamma_{2}^{\prime}},
$$

while Equation (52) becomes:

$$
\frac{a_{1}}{(r+1) \Gamma_{2}^{\prime 2}} J_{4}=0
$$

with:

$$
J_{4} \equiv h c_{1} \Gamma_{2}^{\prime \prime}-\left(h+u h_{u}\right) \Gamma_{2}{ }^{2}+c_{1} h_{v} \Gamma_{2}{ }^{\prime} .
$$

We observe that if the functions $\Gamma_{2}$ and $h$ do not satisfy the equation $J_{4}=0$, we do not obtain the extension of the principal Lie algebra. Then, in order to have extensions of the principal algebra, the functions $\Gamma_{2}$ and $h$ must satisfy the equation $J_{4}=0$. In this case, we obtain the following additional generator:

$$
X_{3}=x \partial_{x}+\frac{1}{r+1} t \partial_{t}+\frac{1}{r+1} u \partial_{u}-\frac{c_{1}}{(r+1) \Gamma_{2}^{\prime}} \partial_{v} .
$$

Summarizing, we obtained six subclasses of class (28), which admit a three-dimensional Lie algebra.

1. $f=f_{0} u^{r}$ with $r \neq-1, \Gamma_{1}(u)=\frac{c_{1} u^{1+r}}{1+r}-\frac{\gamma_{0} u}{r}+c_{2}$ with $\gamma_{0} \neq 0$, the functions $h$ and $\Gamma_{2}$ linked from the following relation:

$$
h(1+r)\left(c_{2}+\Gamma_{2}\right) \Gamma_{2}^{\prime \prime}-\left(h(1+2 r)-u h_{u}\right) \Gamma_{2}{ }^{2}+(1+r)\left(h_{v}-\gamma_{0}\right)\left(c_{2}+\Gamma_{2}\right) \Gamma_{2}{ }^{\prime}=0 .
$$

2. $f=f_{0} u^{r}$ with $r \neq-1, \Gamma_{1}(u)=\frac{c_{1} u^{1+r}}{1+r}+c_{2}$ and the functions $h$ and $\Gamma_{2}$ linked from the following relation:

$$
h(1+r)\left(c_{2}+\Gamma_{2}\right) \Gamma_{2}{ }^{\prime \prime}-\left(h(1+2 r)-u h_{u}\right) \Gamma_{2}{ }^{2}+(1+r)\left(h_{v}\right)\left(c_{2}+\Gamma_{2}\right) \Gamma_{2}{ }^{\prime}=0 .
$$

3. $f=\frac{f_{0}}{u}, \Gamma_{1}(u)=c_{1} \ln (u)+\gamma_{0} u+c_{2}$ with $\gamma_{0} \neq 0$ and the functions $h$ and $\Gamma_{2}$ linked from the following relation:

$$
h c_{1} \Gamma_{2}^{\prime \prime}-\left(h+u h_{u}\right) \Gamma_{2}^{\prime 2}+c_{1}\left(h_{v}-\gamma_{0}\right) \Gamma_{2}^{\prime}=0 .
$$

4. $f=\frac{f_{0}}{u}, \Gamma_{1}(u)=c_{1} \ln (u)+c_{2}$ and the functions $h$ and $\Gamma_{2}$ linked from the following relation:

$$
h c_{1} \Gamma_{2}^{\prime \prime}-\left(h+u h_{u}\right) \Gamma_{2}^{\prime 2}+c_{1}\left(h_{v}\right) \Gamma_{2}^{\prime}=0 .
$$

5. $f=f_{0} u^{s}$ with $s \neq r, 2 r+1, \Gamma_{1}(u)=c_{1}+c_{2} u^{1+2 r-s}$ and the functions $h$ and $\Gamma_{2}$ linked from the following relation:

$$
(1+2 r-s)\left(c_{1}+\Gamma_{2}\right)\left(h \Gamma_{2}^{\prime \prime}+h_{v} \Gamma_{2}^{\prime}\right)+\left(u h_{u}-h(1+4 r-2 s)\right) \Gamma_{2}^{\prime 2}=0 .
$$

6. $f=f_{0} u^{2 r+1}, \Gamma_{1}(u)=c_{1} \ln (u)+c_{2}$ and the functions $h$ and $\Gamma_{2}$ linked from the following relation:

$$
h c_{1} \Gamma_{2}^{\prime \prime}-\left(h+u h_{u}\right) \Gamma_{2}{ }^{2}+c_{1} h_{v} \Gamma_{2}^{\prime}=0 .
$$




\subsection{A Special Case}

In agreement with some news about the biological compatibility of the form of $g$ derived from some previous papers (see, e.g., [29,30] and references insides), in this subsection, we show an example of the application of the previous results.

By selecting the case 1 a from the obtained cases and assuming $r=1$ and $c_{2}=0$ in (56), we consider $f, \Gamma_{1}$, of the following form:

$$
f=f_{0} u, \quad \Gamma_{1}(u)=\gamma_{1} u^{2}-\gamma_{0} u
$$

with $f_{0}, \gamma_{0}, \gamma_{1}$, arbitrary constants. Moreover, we assume:

$$
\Gamma_{2}(v)=\gamma_{2} v+\gamma_{3}
$$

with $\gamma_{2}, \gamma_{3}$, arbitrary constants.

In this case, in order to have an extension on the principal algebra, the function $h(u, v)$ must satisfy the equation $J_{1}=0$, that is:

$$
\left(u h_{u}-3 h\right) \gamma_{2}^{2}+2\left(h_{v}-\gamma_{0}\right)\left(\gamma_{2} v+\gamma_{3}\right) \gamma_{3}=0
$$

Solutions of this equation are functions $h(u, v)$ of the form:

$$
h(u, v)=u^{3} H(\sigma)-2 \frac{\gamma_{0}}{\gamma^{2}}\left(\gamma_{2} v+\gamma_{3}\right),
$$

where $H$ is an arbitrary function of $\sigma=\frac{\gamma_{2} v+\gamma_{3}}{\gamma_{2} u^{2}}$. By assuming $H(\sigma)=\sigma$ in agreement with $[13,29,30]$, we get:

$$
h(u, v)=\frac{\gamma_{2} v+\gamma_{3}}{\gamma_{2}}\left(u-2 \gamma_{0}\right)
$$

The system (28) becomes:

$$
\left\{\begin{array}{l}
u_{t}=f_{0} u_{x}^{2}+f_{0} u u_{x x}+u u_{x}+\gamma_{1} u^{2}-\gamma_{0} u+\gamma_{2} v+\gamma_{3} \\
v_{t}=\frac{\gamma_{2} v+\gamma_{3}}{\gamma_{2}}\left(u-2 \gamma_{0}\right) .
\end{array}\right.
$$

While the third generator is obtained by specializing generator (61) and has the form:

$$
X_{3}=e^{\gamma_{0} t} \partial_{t}-\gamma_{0} e^{\gamma_{0} t} u \partial_{u}-\frac{2 \gamma_{0}}{\gamma_{2}}\left(\gamma_{2} v+\gamma_{3}\right) e^{\gamma_{0} t} \partial_{v}
$$

By considering the generator $k X_{2}+X_{3}$, we get:

$$
u(t, x)=U(z) e^{-\gamma_{0} t}, v(t, x)=\frac{V(z) e^{-2 \gamma_{0} t}-\gamma_{3}}{\gamma 2},
$$

with $z=\frac{\gamma_{0} x}{k}+e^{-\gamma_{0} t}$, while the functions $U(z), V(z)$ are solutions of the reduced system:

$$
\begin{aligned}
& U^{\prime \prime} U f_{0} \gamma_{0}^{2}+U^{\prime 2} f_{0} \gamma_{0}^{2}+k \gamma_{0}(U+k) U^{\prime}+k^{2} V+\gamma_{1} k^{2} U^{2}=0, \\
& \gamma_{0} V^{\prime}+U V=0
\end{aligned}
$$


This reduced system, as well as other cases of biological specializations, will be studied in later research.

Of course, the systems studied here cannot be considered, strictu sensu, as mathematical models. In fact, their constitutive parameters need to be characterized carefully from the biological point view. However, having in mind some previous models concerned with Aedes aegypti [13,14,29-31], we try to stress some structural features of the system (98). To this aim, we rewrite system (98) as:

$$
\left\{\begin{array}{l}
u_{t}=\left(f_{0} u u_{x}\right)_{x}+u u_{x}+\gamma_{1} u\left(u-\frac{\gamma_{0}}{\gamma_{1}}\right)+\gamma_{2}\left(v+\frac{\gamma_{3}}{\gamma_{2}}\right) \\
v_{t}=\left(u-2 \gamma_{0}\right)\left(v+\frac{\gamma_{3}}{\gamma_{2}}\right)
\end{array}\right.
$$

It is easy to ascertain a weak interaction of the equation for the aquatic population on the equation for the winged population. Moreover, in this last one appears a growth for population $u$ having a logistic structure. By identifying $\gamma_{1}$ as a positive rate of maturation of the aquatic forms in winged female mosquitoes and $\gamma_{0}$ as the positive winged population mortality, it is possible to find a threshold value $u_{t r s}=\frac{\gamma_{0}}{\gamma_{1}}$. Finally, the aquatic population evolution equation shows a growth rate $u-2 \gamma_{0}$ ruled by the density of mosquitoes and their mortality.

Remark 1. It is a simple matter to ascertain that the system (103) admits as the special solution:

$$
u=\frac{\gamma_{0}}{\gamma_{1}}, \quad v=-\frac{\gamma_{3}}{\gamma_{2}}
$$

Moreover, it is possible to get other solutions by assuming $v=-\frac{\gamma_{3}}{\gamma_{2}}$, while $u$ is obtained as a solution of equation:

$$
u_{t}=\left(f_{0} u u_{x}\right)_{x}+u u_{x}+\gamma_{1} u\left(u-\frac{\gamma_{0}}{\gamma_{1}}\right) .
$$

For the interested reader, it could be worthwhile noticing that Equation (105) is a particular case of equation considered in [8,9]. Moreover, we can get the results obtained in [8,9] (see Table 1, Case 8 of both papers) by projection. Indeed, the Lie symmetry generator (99) projected in the space $\left(t, x, u, v=-\frac{\gamma_{3}}{\gamma_{2}}\right)$ becomes the Lie symmetry $T_{1}$ of $[8,9]$.

\section{Conclusions}

In this paper, we have considered a class of advection reaction diffusion systems of interest in biomathematics. After having recalled a weak equivalence generator, obtained in a previous work [12], we find some particular cases of the nonlinear system (28) admitting three-dimensional Lie algebras by using a specialization of a projection theorem [24,28]. In this subclass, the constitutive equation characterizing $g$ is assigned as:

$$
g=u^{r} u_{x}+\Gamma_{1}(u)+\Gamma_{2}(v)
$$

that generalizes [12]:

$$
g=\rho u^{r} u_{x}^{s}+\Gamma_{1} u^{a}+\Gamma_{2} v^{b}
$$


where the constants $\rho(\neq 0), \Gamma_{1}, \Gamma_{2}, r, s, a$ and $b$ are constitutive parameters. We derive the principal Lie algebra and the functions admitting at least an extension by one. These results are summarized at the end of Section 3.1. A special case is considered in Section 3.2.

\section{Acknowledgments}

The authors wish to thank the reviewers for their interesting observations and the editor for his very useful comments. Mariano Torrisi was supported from Gruppo Nazionale per la Fisica Matematica of Istituto Nazionale di Alta Matematica.

\section{Author Contributions}

The authors contributed equally to this work.

\section{Conflicts of Interest}

The authors declare no conflict of interest.

\section{References}

1. Cardile, V.; Torrisi, M.; Tracinà, R. On a reaction-diffusion system arising in the development of Bacterial Colonies. In Proceedings of the 10th International Conference in Modern Group Analysis, Larnaca, Cyprus, 24-31 October 2004; Volume 32, p. 38.

2. Medvedev, G.S.; Kaper, T.J.; Kopell, N. A reaction diffusion system with periodic front Dynamics. SIAM J. Appl. Math. 2000, 60, 1601-1638.

3. Torrisi, M.; Tracinà, R. On a class of reaction diffusion systems: Equivalence transformations and symmetries. In Asymptotic Methods in Nonlinear Wave Phenomena; Ruggeri, T., Sammartino, M., Eds.; World Science Publishing Co. Pte. Ltd.: Singapore, 2007; pp. 207-216.

4. Torrisi, M.; Tracinà, R. Exact solutions of a reaction-diffusion system for Proteus Mirabilis bacterial colonies. Nonlinear Anal. Real World Appl. 2011, 12, 1865-1874.

5. Cherniha, R.; Serov, M. Nonlinear systems of the burgers-type equations: Lie and Q-conditional symmetries, ansätze and solutions. J. Math. Anal. Appl. 2003, 282, 305-328.

6. Cherniha, R.; Wilhelmsson, H. Symmetry and exact solution of heat-mass transfer equations in thermonuclear plasma. Ukr. Math. J. 1996, 48, 1434-1449.

7. Cherniha, R.; Serov, M. Lie and non-Lie symmetries of nonlinear diffusion equations with convection term. Symmetry Nonlinear Math. Phys. 1997, 2, 444-449.

8. Cherniha, R.; Serov, M. Symmetries, ansätze and exact solutions of nonlinear second-order evolution equations with convection terms. Eur. J. Appl. Math. 1998, 9, 527-542.

9. Cherniha, R.; Serov, M. Symmetries, ansätze and exact solutions of nonlinear second-order evolution equations with convection terms, II. Eur. J. Appl. Math. 2006, 17, 597-605.

10. Ibragimov, N.H.; Torrisi, M.; Valenti, A. Preliminary group classification of equation $v_{t t}=f\left(x, v_{x}\right) v_{x x}+g\left(x, v_{x}\right)$. J. Math. Phys. 1991, 32, 2988-2995. 
11. Ovsiannikov, L.V. Group Analysis of Differential Equations; Academic Press: New York, NY, USA, 1982.

12. Freire, I.L.; Torrisi, M. Weak equivalence transformations for a class of models in biomathematics. Abstr. Appl. Anal. 2014, doi:10.1155/2014/546083.

13. Freire, I.L.; Torrisi, M. Symmetry methods in mathematical modeling Aedes aegypti dispersal dynamics. Nonlinear Anal. Real World Appl. 2013, 14, 1300-1307.

14. Freire, I.L.; Torrisi, M. Similarity solutions for systems arising from an Aedes aegypti model. Commun. Nonlinear Sci. Numer. Simul. 2014, 19, 872-879.

15. Romano, V.; Torrisi, M. Application of weak equivalence transformations to a group analysis of a drift-diffusion model. J. Phys. A Math. Gen. 1999, 32, 7953-7963.

16. Torrisi, M.; Tracinà, R. Equivalence transformations and symmetries for a heat conduction model. Int. J. Non-Linear Mech. 1998, 33, 473-487.

17. Gazeau, J.P.; Winternitz, P. Symmetries of variable-coefficient Korteweg-de Vries equations. J. Math. Phys. 1992, 33, 4087-4102.

18. Winternitz, P.; Gazeau, J.P. Allowed transformations and symmetry classes of variable coefficient Korteweg-de Vries equations. Phys. Lett. A 1992, 167, 246-250.

19. Akhatov, I.S.H.; Gazizov, R.K.; Ibragimov, N.H. Nonlocal symmetries. Heuristic approach. J. Sov. Math. 1991, 55, 1401-1450.

20. Lisle, I.G. Equivalence Transformation for Classes of Differential Equations. Ph.D. Thesis, University of British Columbia, Vancouver, BC, Canada, 1992.

21. Khalique, C.M.; Mahomed, F.M.; Ntsime, B.P. Group classification of the generalized Emden-Fowler-type equation. Nonlinear Anal. Real World Appl. 2009, 10, 3387-3395.

22. Ibragimov, N.H. CRC Handbook of Lie Group Analysis of Differential Equations; CRC Press: Boca Raton, FL, USA, 1996.

23. Molati, M.; Khalique, C.M. Lie group classification of a generalized Lane-Emden Type system in two dimensions. J. Appl. Math. 2012, doi:10.1155/2012/405978.

24. Torrisi, M.; Tracinà, R. Equivalence transformations for systems of first order quasilinear partial differential equations. In Modern Group Analysis VI: Developments in Theory, Computation and Application; New Age International(P) Ltd.: New Delhi, India, 1996; pp. 115-135.

25. Torrisi, M.; Tracinà, R.; Valenti, A. Group analysis approach for a non linear differential system arising in diffusion phenomena. J. Math. Phys. 1996, 37, 4758-4767.

26. Gambino, G.; Greco, A.M.; Lombardo, M.C. A group analysis via weak equivalence transformations for a model of tumour encapsulation. J. Phys. A 2004, 37, 3835-3846.

27. Ibragimov, N.H.; Säfström, N. The equivalence group and invariant solutions of a tumour growth model. Commun. Nonlinear Sci. Num. Simul. 2004, 9, 61-69.

28. Ibragimov, N.H.; Torrisi, M. A simple method for group analysis and its application to a model of detonation. J. Math. Phys. 1992, 33, 3931-3937.

29. Maidana, N.A.; Yang, H.M. Describing the geographic spread of dengue disease by traveling waves. Math. Biosci. 2008, 215, 64-77. 
30. Takahashi, L.T.; Maidana, N.A.; Ferreira, W.C., Jr.; Pulino, P.; Yang, H.M. Mathematical models for the Aedes aegypti dispersal dynamics: Traveling waves by wing and wind. Bull. Math. Biol. 2005, 67, 509-528.

31. Bacani, F.; Freire, I.L.; Maidana, N.A.; Torrisi, M. Modelagem para a dinâmica populacional do Aedes aegypti via simetrias de Lie. Proc. Ser. Braz. Soc. Appl. Comput. Math. 2015, 3, doi:10.5540/03.2015.003.01.0055.

(c) 2015 by the authors; licensee MDPI, Basel, Switzerland. This article is an open access article distributed under the terms and conditions of the Creative Commons Attribution license (http://creativecommons.org/licenses/by/4.0/). 